\title{
Article \\ Bimetallic ZIF-Derived Co/N-Codoped Porous Carbon Supported Ruthenium Catalysts for Highly Efficient Hydrogen Evolution Reaction
}

\author{
Hui Qi ${ }^{\dagger}$, Xinglong Guan ${ }^{\dagger}$, Guangyu Lei, Mengyao Zhao, Hongwei He, Kai Li, Guoliang Zhang, Fengbao Zhang, \\ Xiaobin Fan, Wenchao Peng (1) and Yang Li * (i)
}

check for

updates

Citation: Qi, H.; Guan, X.; Lei, G.; Zhao, M.; He, H.; Li, K.; Zhang, G.; Zhang, F.; Fan, X.; Peng, W.; et al. Bimetallic ZIF-Derived Co/N-Codoped Porous Carbon Supported Ruthenium Catalysts for Highly Efficient Hydrogen Evolution Reaction. Nanomaterials 2021, 11, 1228.

https://doi.org/10.3390/nano11051228

Academic Editor: Akinobu

Yamaguchi

Received: 11 April 2021

Accepted: 28 April 2021

Published: 6 May 2021

Publisher's Note: MDPI stays neutral with regard to jurisdictional claims in published maps and institutional affiliations.

Copyright: (c) 2021 by the authors. Licensee MDPI, Basel, Switzerland. This article is an open access article distributed under the terms and conditions of the Creative Commons Attribution (CC BY) license (https:// creativecommons.org/licenses/by/ $4.0 /)$.
Lab of Advanced Nano-Structure and Transfer Process, Department of Chemical Engineering, Tianjin University, Tianjin 300354, China; qihui1101@tju.edu.cn (H.Q.); tjugxl@tju.edu.cn (X.G.); guangyulei@tju.edu.cn (G.L.); Zhaomy@tju.edu.cn (M.Z.); hehongwei@tju.edu.cn (H.H.); tju-likai@tju.edu.cn (K.L.); zhangguoliang@tju.edu.cn (G.Z.); fbzhang@tju.edu.cn (F.Z.); xiaobinfan@tju.edu.cn (X.F.); wenchao.peng@tju.edu.cn (W.P.)

* Correspondence: liyang1895@tju.edu.cn; Tel.: +86-22-2789-0090

+ The authors contribute equal to this study.

\begin{abstract}
Exploring the economical, powerful, and durable electrocatalysts for hydrogen evolution reaction (HER) is highly required for practical application. Herein, nanoclusters-decorated ruthenium, cobalt nanoparticles, and nitrogen codoped porous carbon (Ru-pCo@NC) are prepared with bimetallic zeolite imidazole frameworks (ZnCo-ZIF) as the precursor. Thus, the prepared Ru-pCo@NC catalyst with a low Ru loading of $3.13 \mathrm{wt} \%$ exhibits impressive HER catalytic behavior in $1 \mathrm{M} \mathrm{KOH}$, with an overpotential of only $30 \mathrm{mV}$ at the current density of $10 \mathrm{~mA} \mathrm{~cm}{ }^{-2}$, Tafel slope as low as $32.1 \mathrm{mV} \mathrm{dec}^{-1}$, and superior stability for long-time running with a commercial $20 \mathrm{wt} \% \mathrm{Pt} / \mathrm{C}$. The excellent electrocatalytic properties are primarily by virtue of the highly specific surface area and porosity of carbon support, uniformly dispersed Ru active species, and rapid reaction kinetics of the interaction between $\mathrm{Ru}$ and $\mathrm{O}$.
\end{abstract}

Keywords: bimetallic ZIF; porous carbon; ruthenium; hydrogen evolution reaction

\section{Introduction}

Among the promising clean energy sources, hydrogen has attracted intense investigation for its high energy density and zero emission (no greenhouse gases are emitted) [1-3]. Electrocatalytic water splitting to generate ultrapurity and pollution-free hydrogen is an effective and sustainable approach in the industry $[4,5]$. As a critical component of water electrolysis technology, hydrogen evolution reaction (HER) at the cathode urgently demands highly efficient and durable catalysts for large-scale application [6]. Until now, platinum (Pt)-based catalysts are constantly at the leading edge of most HER electrocatalysts $[7,8]$. However, the widespread development and application of Pt-based catalysts are held up because of the insufficient on-earth storage and high cost of Pt. Moreover, there is also an issue linked to the stability of Pt-based electrocatalysts in strong alkaline and acidic electrolytes [9]. Hence, developing other low-precious metal catalysts for the substitution of Pt has attracted more and more interest in recent years [10-12]. Ruthenium $(\mathrm{Ru})$ is known to be the cheapest Pt-group metal, which just costs $\sim 4 \%$ of Pt, yet it possesses a resembling metal-hydrogen binding strength $\left(\mathrm{M}-\mathrm{H}^{*}\right)$ to $\mathrm{Pt}\left(\sim 65 \mathrm{kcal} \mathrm{mol}^{-1}\right)$ and a stronger corrosion resistance $[13,14]$. Currently, numerous Ru-based catalysts have been designed and have shown pleasant catalytic behaviors for HER. For example, Chang et al. prepared the pure $\mathrm{RuP}_{x}$ catalysts via a simple and controllable heat-treatment method, which exhibited impressive catalytic performance for $\mathrm{pH}$-universal HER [15]. Zong et al. reported the core-shelled Fe@Ru nanoparticles (4.8 wt\% Ru) enclosed in N-doped carbon 
nanoframes, which presented an overpotential of $55 \mathrm{mV}$ at $10 \mathrm{~mA} \mathrm{~cm}{ }^{-2}\left(\eta_{10}\right)$ and a Tafel slope of $77.3 \mathrm{mV} \mathrm{dec}^{-1}$ for alkaline HER [16].

Finding a suitable material as the support for active centers is also crucial to ensure the effective interactions between the different phases involved in most electrocatalytic reaction processes $[17,18]$. Recently, the preparation of metal/carbon-based porous materials using metal organic frameworks (MOFs) as precursors has emerged as a hot research issue $[4,19,20]$. Zeolite imidazole frameworks (ZIFs), as a new type of MOFs with zeolitelike 3D topological structure, are considered promising to derive nitrogen-rich carbon with highly specific surface area (SSA), abundant porosity, and good structural stability [21,22]. Among them, zinc (Zn)-based ZIF-8 can afford highly microporous nitrogen-doped carbons with large SSA and high porosity after thermal activation, whereas it cannot provide wellgraphitized carbon, leading to low electrical conductivity [23]. On the other hand, cobalt (Co)-based ZIF-67 can offer enhanced electrical conductivity due to the catalytic graphitization by Co nanoparticles (NPs) but causes the loss of SSA and porosity [24]. Hence, if the two are combined, high SSA and conductivity can be achieved at the same time.

In the view of the above considerations, a highly active electrocatalyst by introducing $\mathrm{Ru}$ nanoclusters into the $\mathrm{Co} / \mathrm{N}$-codoped porous carbon born of a bimetallic ZIF (ZnCo-ZIF) is reported (denoted as Ru-pCo@NC) in this study. Benefiting from the good electrical conductivity, large SSA, uniformly dispersed active sites, and rapid kinetics induced by the interaction between Ru and O, Ru-pCo@NC with low Ru loading content (3.13 wt\%) display impressing catalytic properties toward HER in $1 \mathrm{M} \mathrm{KOH}$, with $\eta_{10}$ of $30 \mathrm{mV}$, Tafel slope of $32.1 \mathrm{mV} \mathrm{dec}^{-1}$, and high durability, rivaling commercial $20 \mathrm{wt} \% \mathrm{Pt} / \mathrm{C}$ and outperforming most Ru-based electrocatalysts that have been studied (Table S1).

\section{Materials and Methods}

\subsection{Materials}

All chemicals are commercial and used without further purification. Zinc (II) nitrate hexahydrate $\left(\mathrm{Zn}\left(\mathrm{NO}_{3}\right)_{2} \cdot 6 \mathrm{H}_{2} \mathrm{O}\right)$, cobalt (II) nitrate hexahydrate $\left(\mathrm{Co}\left(\mathrm{NO}_{3}\right)_{2} \cdot 6 \mathrm{H}_{2} \mathrm{O}\right)$ and methanol $\left(\mathrm{CH}_{4} \mathrm{O}\right)$ were purchased from Kermel Chemical Reagent Co. Ltd. (Tianjin, China). 2-methylimidazole $\left(\mathrm{C}_{4} \mathrm{H}_{6} \mathrm{~N}_{2}\right)$ and commercial $\mathrm{Pt} / \mathrm{C}(20 \mathrm{wt} \%)$ were purchased from Macklin Biochemical Co. Ltd. (Shanghai, China). Ruthenium (III) chloride $\left(\mathrm{RuCl}_{3}\right)$ was purchased from Heowns Biochemical Technology Co. Ltd. (Tianjin, China). Nafion solution $(5 \%)$ and potassium hydroxide $(\mathrm{KOH})$ were purchased from Sigma-Aldrich Co. Ltd. (Shanghai, China).

\subsection{Synthesis of ZIF Precursors}

For the synthesis of $\mathrm{ZIF}-67,0.02 \mathrm{~mol} \mathrm{Co}\left(\mathrm{NO}_{3}\right)_{2} \cdot 6 \mathrm{H}_{2} \mathrm{O}$ and $0.08 \mathrm{~mol}$ 2-methylimidazole (2-MeIm) were dissolved in $60 \mathrm{~mL}$ methanol (MeOH) to form two solutions, respectively. Then, the solution of 2-MeIm was added to the solution of $\mathrm{Co}\left(\mathrm{NO}_{3}\right)_{2} \cdot 6 \mathrm{H}_{2} \mathrm{O}$ with vigorous stirring for $24 \mathrm{~h}$ at room temperature. The resulting purple product was collected by centrifugation, washed thoroughly with water and $\mathrm{MeOH}$ for several times, and finally dried in vacuum at $60^{\circ} \mathrm{C}$ overnight.

ZIF-8 was formed by the similar method above except that the $\mathrm{Co}\left(\mathrm{NO}_{3}\right)_{2} \cdot 6 \mathrm{H}_{2} \mathrm{O}$ was replaced by $\mathrm{Zn}\left(\mathrm{NO}_{3}\right)_{2} \cdot 6 \mathrm{H}_{2} \mathrm{O}$.

For the synthesis of $\mathrm{ZnCo}-\mathrm{ZIF}$, the mixture of $0.014 \mathrm{~mol} \mathrm{Zn}\left(\mathrm{NO}_{3}\right)_{2} \cdot 6 \mathrm{H}_{2} \mathrm{O}$ and $0.006 \mathrm{~mol}$ $\mathrm{Co}\left(\mathrm{NO}_{3}\right)_{2} \cdot 6 \mathrm{H}_{2} \mathrm{O}$ were dissolved in $60 \mathrm{~mL} \mathrm{MeOH}$ to form a mixture solution. Then, a solution of $0.08 \mathrm{~mol} 2-\mathrm{MeIm}$ in $60 \mathrm{~mL} \mathrm{MeOH}$ was added to the above mixture solution with vigorous stirring for $24 \mathrm{~h}$ at room temperature. The resulting light-purple product was collected by centrifugation, washed thoroughly with water and $\mathrm{MeOH}$ several times, and finally dried in vacuum at $60^{\circ} \mathrm{C}$ overnight.

\subsection{Synthesis of $p C o @ N C, N C$, and Co@NC}

For the preparation of $\mathrm{pCo} @ \mathrm{NC}$, the as-synthesized ZnCo-ZIF nanocrystals were transferred into a corundum boat and placed in the tube furnace under nitrogen flow. The 
sample was heated to $950{ }^{\circ} \mathrm{C}$ with a heating rate of $5{ }^{\circ} \mathrm{C} \min ^{-1}$ and kept for $2 \mathrm{~h}$. After naturally cooling down to room temperature, the obtained black powder was collected and directly used for further characterization and measurement. A similar method, as described above, was used for the preparation of NC and Co@NC with the precursor of ZIF-8 and ZIF-67, respectively.

\subsection{Synthesis of Ru-pCo@NC}

A total of $50 \mathrm{mg}$ of the as-prepared pCo@NC nanoparticles was dispersed in $20 \mathrm{~mL}$ deionized water with ultrasonic stirring for $1 \mathrm{~h}$ to form a completely homogeneous suspension. Then, $0.048 \mathrm{mmol}$ of $\mathrm{RuCl}_{3}$ was added to the above suspension. After reacting at $40{ }^{\circ} \mathrm{C}$ for $24 \mathrm{~h}$ in the shaker, the solid was collected, washed thoroughly with water and $\mathrm{MeOH}$ for several times, and finally dried in vacuum at $60{ }^{\circ} \mathrm{C}$ overnight. For comparison, a series of composites with $0.024,0.096$, and $0.144 \mathrm{mmol}$ of $\mathrm{RuCl}_{3}$ were also obtained and denoted as Ru-pCo@NC-1, Ru-pCo@NC-2, and Ru-pCo@NC-3.

\subsection{Characterization}

The composition and structure of the samples were observed by X-ray diffraction (XRD) instrument ( $\mathrm{Cu} \mathrm{K} \alpha$ radiation) and Raman spectrometer. The surface morphologies and microscopic structure were characterized by scanning electron microscopy (SEM), highresolution transmission electron microscopy (TEM), high-angle annular dark field-scanning transmission electron microscopy (HAADF-STEM), and energy dispersive X-ray (EDX) spectroscopy. $\mathrm{N}_{2}$ adsorption/desorption measurements were carried out for SSA and pore size distribution. Further composition and valence state information were obtained by X-ray photoelectron spectroscopy (XPS).

\subsection{Electrochemical Measurements}

The electrochemical measurements were conducted on a $\mathrm{CHI} 660 \mathrm{E}$ electrochemical workstation with a typical three-electrode setup in $1 \mathrm{M} \mathrm{KOH}$ electrolytes at about $25{ }^{\circ} \mathrm{C}$. The catalyst coated on carbon fiber cloth was used as the working electrode, an $\mathrm{Ag} / \mathrm{AgCl}$ electrode in saturated $\mathrm{KCl}$ as the reference electrode, and a graphite rod as the counter electrode. The working electrode was prepared as follows: $5.0 \mathrm{mg}$ of the catalyst was dispersed in the mixture of distilled water $(0.45 \mathrm{~mL})$ and Nafion $(5 \mathrm{wt} \%, 0.05 \mathrm{~mL})$ and ultrasonicated for $1 \mathrm{~h}$ to form a uniform suspension. Then, $50 \mu \mathrm{L}$ of the suspension was dropped onto a piece of clean carbon fiber cloth $\left(0.5 \mathrm{~cm}^{2}\right)$ and dried at room temperature.

The linear sweep voltammetry (LSV) curves were collected from -0.8 to $-1.8 \mathrm{~V}$ (vs. $\mathrm{Ag} / \mathrm{AgCl}$ ) with a scan rate of $5 \mathrm{mV} \mathrm{s}^{-1}$. All potentials reported in this work were converted to the reversible hydrogen electrode (RHE) scale according to $E(\mathrm{RHE})=E(\mathrm{Ag} / \mathrm{AgCl})$ $+0.059 \mathrm{pH}+0.197\left(25^{\circ} \mathrm{C}\right)$. In addition, All polarization curves were corrected by the $i R$ contribution within the system following the equation of $\mathrm{E}$ ( $i R$ corrected) $=\mathrm{E}(\mathrm{RHE})-$ $i R$, where $i$ is the current and $R$ is the solution resistance measured by electrochemical impedance spectroscopy (EIS) measurements. EIS was examined under alternating-current voltage amplitude of $5 \mathrm{mV}$ with a frequency from 0.01 to $10^{6} \mathrm{~Hz}$. The electrochemical double-layer capacitance $\left(\mathrm{C}_{\mathrm{dl}}\right)$ was determined by the cyclic voltammograms $(\mathrm{CVs})$ measured at different scan rates from 20 to $100 \mathrm{mV} \mathrm{s}^{-1}$ in the potential range of 0.1 to $0.3 \mathrm{~V}$ (vs. RHE). The stability tests were carried out through CV method with potential scanning between -0.2 to $-0.04 \mathrm{~V}$ (vs. RHE) at $50 \mathrm{mV} \mathrm{s}^{-1}$ for 10,000 cycles and chronoamperometry at a constant overpotential of $43 \mathrm{mV}$ (vs. RHE).

\section{Results and Discussion}

\subsection{Structural Characterization}

The synthesis schematic diagram of Ru-pCo@NC is outlined in Figure 1. Briefly, the $\mathrm{ZnCo}-\mathrm{ZIF}$ is firstly synthesized by the self-assembly of $\mathrm{Zn}^{2+}$ ions, $\mathrm{Co}^{2+}$ ions (the $\mathrm{Zn} / \mathrm{Co}$ molar ratio $=7: 3)$, and 2-methylimidazole $(2-\mathrm{MeIm})$ ligands at room temperature. Then, the $\mathrm{ZnCo}-\mathrm{ZIF}$ precursor is calcinated to form N-doped porous carbon with metallic Co 
NPs encapsulated and some nanotubes decorated (pCo@NC). Subsequently, low content $\mathrm{Ru}$ species (determined as $3.13 \mathrm{wt} \%$ by inductively coupled plasma-mass spectrometry (ICP-MS)) are loaded onto the carbon skeleton to obtain the Ru-pCo@NC.

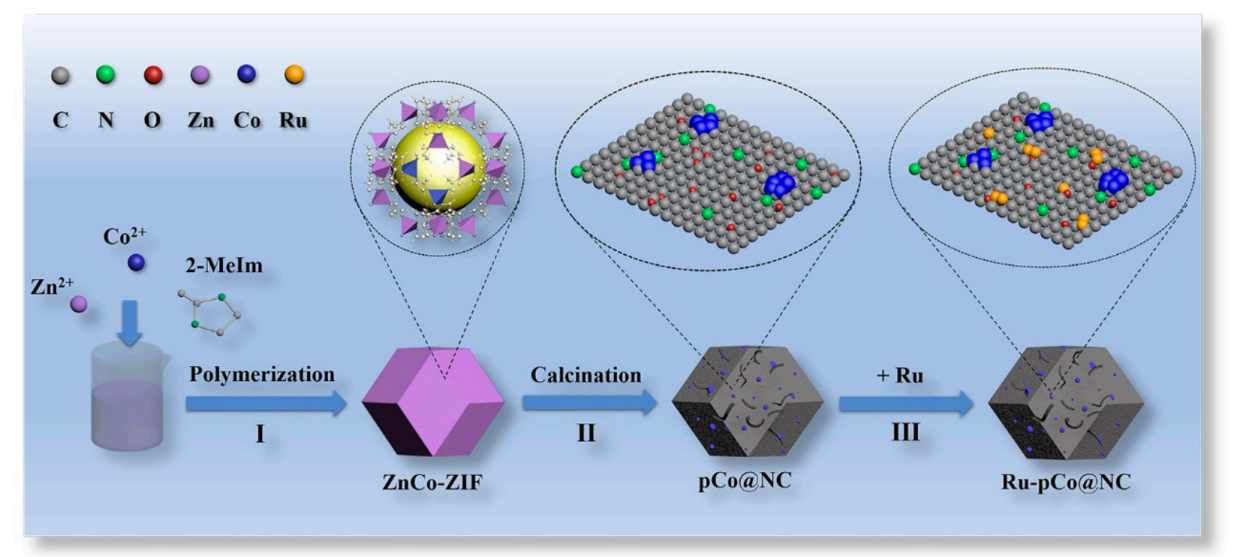

Figure 1. Schematic illustration of the Ru-pCo@NC preparation process.

The XRD patterns of the as-synthesized ZIF-8, ZIF-67, and ZnCo-ZIF are presented in Figure S1a, which are well matched to that of the simulated ZIF-67 due to their similar crystal phase, indicating the ZIF structures are successfully synthesized. For the pattern of pure NC derived from the ZIF-8 (Figure 2a), the two bulging peaks at around $26^{\circ}$ and $44^{\circ}$ belong to the amorphous carbon. For those of Co@NC, pCo@NC, and Ru-pCo@NC, the diffraction peak found at $26.2^{\circ}$ can be designated as the (002) facet of graphitic carbon. Obviously, the presence of Co can improve the graphitization, which is beneficial to the electrical conductivity. Another three prominent peaks at $44.2^{\circ}, 51.5^{\circ}$, and $75.9^{\circ}$ correspond to the (111), (200), and (220) facets of face-centered-cubic (fcc) Co (JCPDS card No. 15-0806), respectively [25]. Notably, no characteristic diffraction peak relevant to Ru species is marked in the XRD pattern of Ru-pCo@NC, most probably owing to their smaller size and low crystallinity [13], as discussed further below. Moreover, the XRD patterns of Ru-pCo@NC-1, Ru-pCo@NC-2, and Ru-pCo@NC-3 composites show almost the same, indicating that the increase in $\mathrm{Ru}$ amount has no effect on their crystal structure (Figure S1b).

The SEM is used for the morphology observation of the samples. As seen in Figure S2a-c, the as-synthesized ZIF-8, ZIF-67, and ZnCo-ZIF are all in distinct rhombic polyhedron shapes with relatively flat surfaces and average size of $\sim 700-800 \mathrm{~nm}$. The obtained NC inherits the overall polyhedron structure of the ZIF-8 precursor (Figure S2d). For Co@NC, plenty of larger-sized Co particles are distributed on the surfaces due to severe agglomeration under the high-temperature pyrolysis condition (Figure S2e). In contrast, there are no apparent large Co NPs formed on the surfaces of pCo@NC, because the introduction of $\mathrm{Zn}$ act as a spacer between the Co sites in the ZnCo-ZIF precursor, and $\mathrm{Zn}$ species will evaporate during the carbonization process [26], thus preventing the further aggregation of Co. Moreover, the surfaces of $\mathrm{pCo} @ \mathrm{NC}$ are decorated with some curved carbon nanotubes (CNTs) (Figure S2f), which should be converted from the organic skeleton by the catalysis of Co NPs [27]. The morphological features of Ru-pCo@NC are revealed in Figure 2b, and there is no noticeable change compared with that of pCo@NC, indicating that the addition of Ru nearly has no effect on the morphology of pCo@NC support. In the TEM image of Ru-pCo@NC (Figure 2c), many metal nanoparticles with a diameter range of $5 \sim 40 \mathrm{~nm}$ are homogeneously embedded in the carbon matrix. Moreover, for the HRTEM image of Ru-pCo@NC (insert of Figure 2c), the distinct lattice spaces of the particle are measured to be 0.204 and $0.177 \mathrm{~nm}$, corresponding to Co (111) and (200) planes of the cubic phase, respectively. Abundant graphitic carbon layers can be observed around the Co NPs and show the interplanar spacing of the carbon (002) crystal plane $(0.34 \mathrm{~nm})$. The outer graphite layers could availably prevent the accumulation of Co NPs, thus improving 
the activity and durability of catalyst in the electrochemical test. Additionally, the direct interfacial interaction between the Co NPs and outer carbon layers would enhance electrical conductivity and favor electron transfer [28]. Moreover, no definite space lattice of Ru species is found, which coincides with the result of XRD analysis and further proves the low crystallinity of Ru. In Figure 2d, the HAADF-STEM and corresponding EDX elemental mapping spectroscopy of Ru-pCo@NC corroborate the existence of C, N, O, Co, and Ru in the composite, from which it can be seen that Ru species are homogeneously dispersed on the support without obvious aggregation. Therefore, it is referred that Ru exists in the form of smaller nanoclusters.
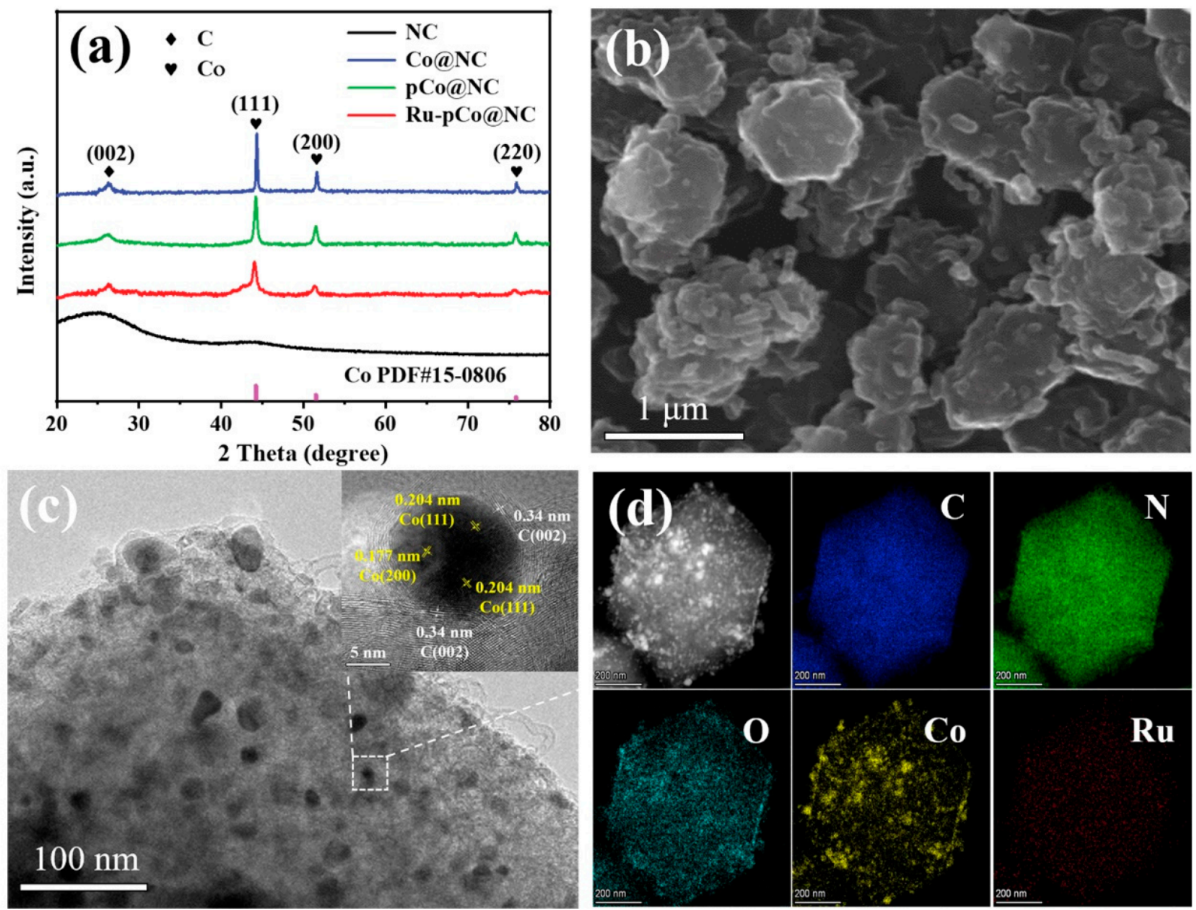

Figure 2. (a) XRD patterns of NC, Co@NC, pCo@NC, and Ru-pCo@NC. (b) SEM image, (c) TEM image and HRTEM image (insert), and (d) HAADF-STEM and corresponding EDX elemental mapping images of Ru-pCo@NC.

The elemental composition and electron states of Ru-pCo@NC are detected by XPS. The survey spectra of pCo@NC and Ru-pCo@NC in Figure S3a disclose C, N, O, and Co elements in both samples and additional Ru element in Ru-pCo@NC. As can been seen in the high-resolution XPS spectrum of Zn 2p in Ru-pCo@NC (Figure S3b), the Zn signal is hardly detected, and the Zn content is measured to be only $0.12 \%$, confirming the almost evaporation of $\mathrm{Zn}$ species during the high-temperature calcination process. In Figure $3 \mathrm{a}$, the high-resolution spectrum of Ru 3d in Ru-pCo@NC, which partially overlaps with C 1s, can be deconvoluted into $\mathrm{Ru} 3 \mathrm{~d}_{5 / 2}(281.8 \mathrm{eV})$ and $\mathrm{Ru} 3 \mathrm{~d}_{3 / 2}(286.4 \mathrm{eV})$ of zero-valence $\mathrm{Ru}$ $\left(\mathrm{Ru}^{0}\right)$ [29], along with $\mathrm{C}-\mathrm{C} / \mathrm{C}=\mathrm{C}(284.6 \mathrm{eV}), \mathrm{C}-\mathrm{O} / \mathrm{C}-\mathrm{N}(285.5 \mathrm{eV})$, and $\mathrm{C}=\mathrm{O}(288.4 \mathrm{eV})$ [30]. It is known that the $\mathrm{Ru} 3 \mathrm{p}_{3 / 2}$ and $\mathrm{Ru} 3 \mathrm{p}_{1 / 2}$ peaks of $\mathrm{Ru}^{0}$ generally appear at 462.5 and $484.9 \mathrm{eV}$ [31]. From the Ru 3p spectrum of Ru-pCo@NC (Figure 3b), the Ru 3p $p_{3 / 2}$ and Ru $3 \mathrm{p}_{1 / 2}$ peaks have higher binding energies and locate at 463.1 and $485.6 \mathrm{eV}$, respectively. Significantly, the increase in binding energy means the electron deficiency, implying that there is an electronic effect between $\mathrm{Ru}$ and the surrounding species. On the contrary, a negative shift in binding energy is detected in the $\mathrm{O}$ 1s spectrum of Ru-pCo@NC compared with that of pCo@NC (Figure 3c). In addition, the oxygen species are identified as the Ru-O bond $(529.8 \mathrm{eV}),-\mathrm{OH}(531.3 \mathrm{eV})$, and physically or chemically bonded $\mathrm{H}_{2} \mathrm{O}(533.2 \mathrm{eV})$ on the carbon surface [32] from the $\mathrm{O}$ 1s spectrum of Ru-pCo@NC. Therefore, the increased binding energy of $\mathrm{Ru}$ and the decreased binding energy of $\mathrm{O}$ can arise from the electronic effect between $\mathrm{Ru}$ and $\mathrm{O}$ species on the support, thus resulting in the electron transfer from 
$\mathrm{Ru}$ to $\mathrm{O}$ [33]. As proved by previous studies, Ru has a moderated hydrogen binding energy that was close to that of $\mathrm{Pt}[9,34]$. In our case, the interaction between $\mathrm{Ru}$ and $\mathrm{O}$ should lead to electron redistribution within the composite, thus facilitating the charge transfer and reaction kinetics to produce $\mathrm{Ru}-\mathrm{H}^{*}$ for the HER. The high-resolution $\mathrm{N}$ 1s curve of Ru-pCo@NC is divided into the pyridinic N (398.6 eV), pyrrolic N (400.0 eV), and graphitic $\mathrm{N}(401.1 \mathrm{eV})$ species (Figure 3d) [35]. Some studies have explained that these $\mathrm{N}$ doping can induce changes in the electronic structure of the carbon matrix, thereby also leading to a certain degree of promotion in electrochemical performance of carbon materials $[36,37]$. The deconvoluted spectrum of $\mathrm{Co} 2 \mathrm{p}$ reveals that metallic $\mathrm{Co}\left(\mathrm{Co}^{0}\right)$ and $\mathrm{Co}^{2+}$ species coexist in Ru-pCo@NC (Figure S3c) [11]. The presence of cobalt oxides could be attributed to the exposure of samples in air, which can be explained because the XPS analysis concentrates primarily on the surface chemical elements of a sample (about $\sim 3-5 \mathrm{~nm}$ ) [38].
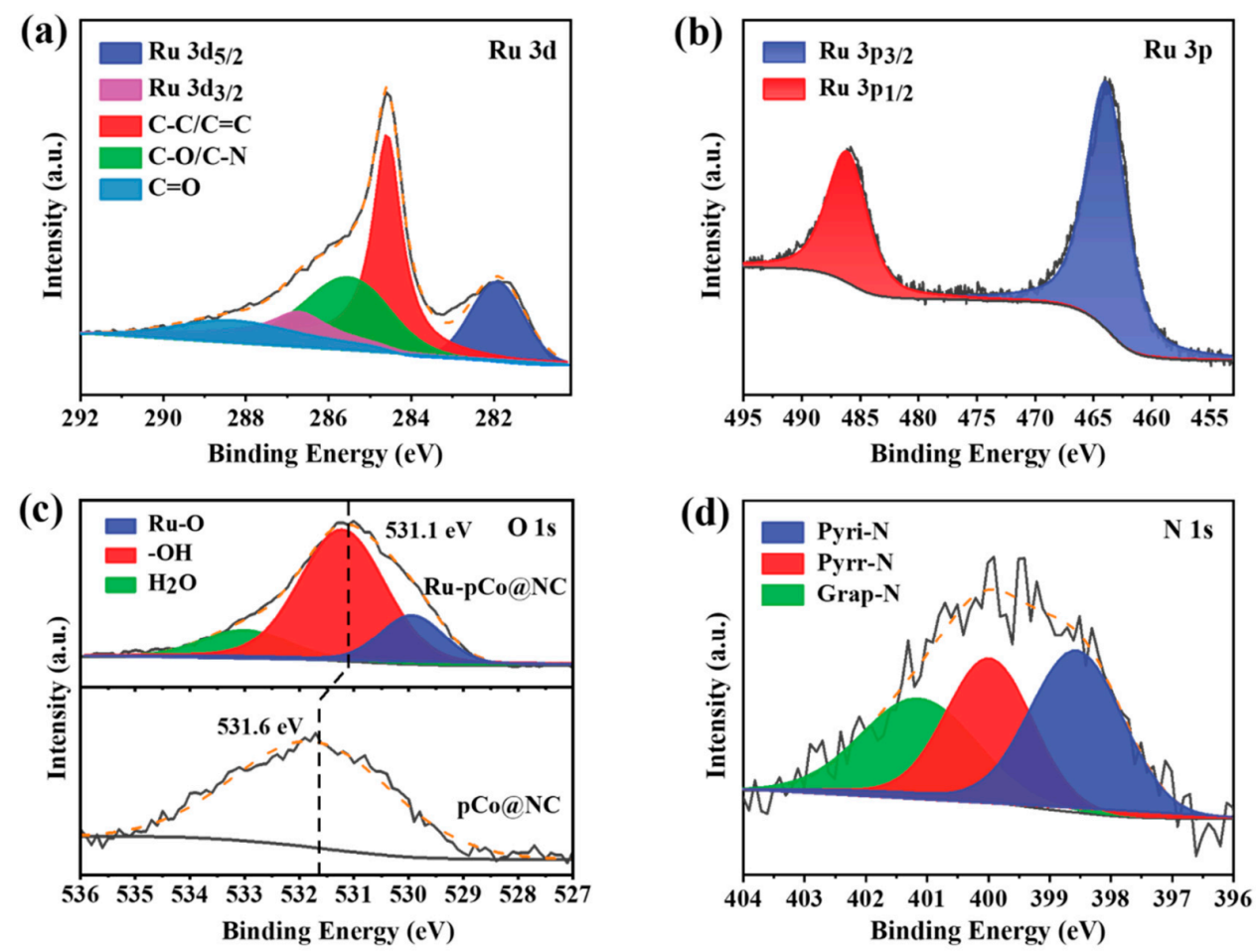

Figure 3. High-resolution XPS spectra of (a) Ru 3d (partially overlaps with C 1s) and (b) Ru 3p of Ru-pCo@NC, (c) O 1s of Ru-pCo@NC and Co@NC, and (d) N 1s of Ru-pCo@NC.

The pore structure and SSA of the samples are analyzed by the $\mathrm{N}_{2}$ adsorption/desorption measurement. Co@NC and pCo@NC both show the type-IV isotherms (Figure S4a), demonstrating the existence of mesopores in the samples. When the relative pressure is low $\left(\mathrm{P} / \mathrm{P}_{0}<0.1\right)$, the adsorption capacity of $\mathrm{pCo} @ \mathrm{NC}$ for $\mathrm{N}_{2}$ is seen as higher than that of Co@NC, signifying more abundant pores in pCo@NC. The SSAs of Co@NC and pCo@NC determined by the Brunauer-Emmett-Teller (BET) method are 132.5 and $428.2 \mathrm{~m}^{2} \mathrm{~g}^{-1}$, respectively (see detailed structure information in Table S2). Obviously, the bimetallic ZnCo-ZIF as a precursor can provide significantly increased porosity and SSA of support owing to the evaporation of $\mathrm{Zn}$ under the high-temperature carbonization condition. The insert of Figure S4a is the pore size distributions of Co@NC and pCo@NC. More importantly, Ru-pCo@NC retains the high SSA of $411.3 \mathrm{~m}^{2} \mathrm{~g}^{-1}$ and mesopore characteristic of pCo@NC (Figure 4a). In addition, the pore size distribution of Ru-pCo@NC (insert of Figure 4a) displays a sharp peak centered at $\sim 3.28 \mathrm{~nm}$. As we all know, a large SSA of electrocatalyst is important to the exposure and accessibility of active species, and mesopores are conducive to the mass transport in the electrocatalytic process and accelerate the reaction rate. Further, the carbon components of these samples are probed by Raman spectroscopy. The two pronounced vibrational peaks at 1350 and $1580 \mathrm{~cm}^{-1}$ are characteristics of the $\mathrm{D}$ and G 
bands for carbon materials (Figure S4b), which can be used as indicators of the defective component and graphitic component, respectively [39]. The intensity ratio of $\mathrm{D}$ band and $\mathrm{G}$ band $\left(I_{D} / I_{G}\right)$ is measured to be 0.94 for pCo@NC, higher than that of Co@NC $\left(I_{D} / I_{G}=0.81\right)$, implying that pCo@NC is less graphitized than $\mathrm{Co} @ \mathrm{NC}$ due to the formation of more pores and defects, and these defect sites have proven helpful for electrochemical catalysis [40]. The $I_{D} / I_{G}$ value of Ru-pCo@NC is calculated to be 0.96 (Figure $4 b$ ), demonstrating that the incorporation of Ru into pCo@NC still retains the advantageous carbon structure of the support.
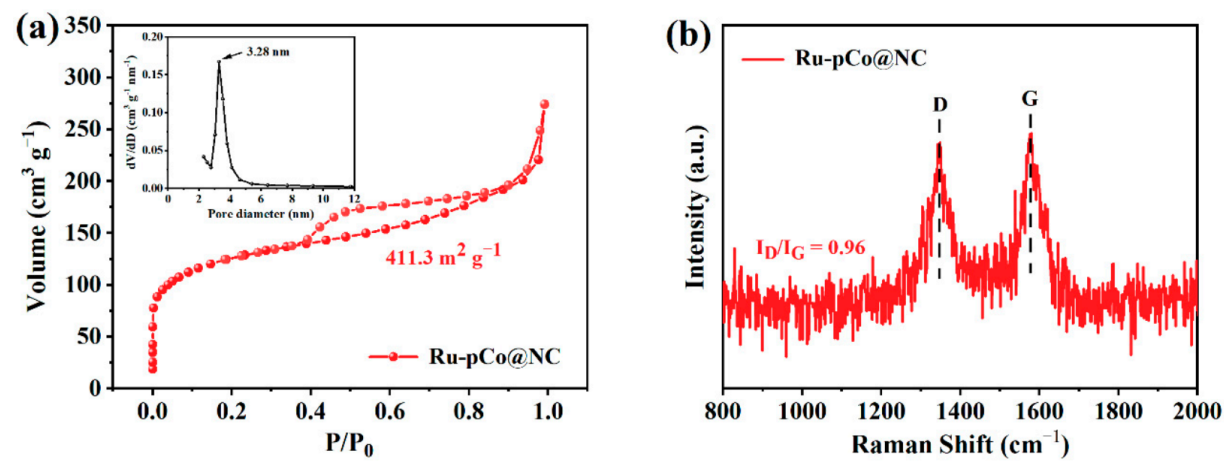

Figure 4. (a) $\mathrm{N}_{2}$ adsorption/desorption isotherms and pore size distribution (insert) and (b) Raman spectrum of Ru-pCo@NC.

\subsection{Electrocatalytic HER Characterization}

The HER catalytic behaviors of the as-obtained catalysts are examined in $1 \mathrm{M} \mathrm{KOH}$ electrolyte. Figure S5 depicts the iR-corrected LSV curves of the Ru-pCo@NC composites with different $\mathrm{Ru}$ amount. Accordingly, the $\eta_{10}$ values are measured to be $55,30,29$, and $29 \mathrm{mV}$ for Ru-pCo@NC-1, Ru-pCo@NC, Ru-pCo@NC-2, and Ru-pCo@NC-3, respectively. It is noteworthy that the catalytic activity initially improves and then remains stable with $\mathrm{Ru}$ amount increasing, which is possibly because the $\mathrm{Ru}$ precursor is excessive. In consideration of cost, the Ru-pCo@NC is selected as the optimum catalyst for further investigation, as described below.

The LSV curves of NC, Co@NC, pCo@NC, Ru-pCo@NC, and commercial 20 wt\% Pt/C are presented in Figure 5a. As observed, the HER catalytic behavior of Co@NC is superior to NC, and pCo@NC delivers an obvious performance improvement $\left(\eta_{10}=207 \mathrm{mV}\right)$, which proves the combined effect of graphitization, SSA, and porosity on HER catalytic activity. Furthermore, great activity promotion is observed for Ru-pCo@NC $\left(\eta_{10}=30 \mathrm{mV}\right)$, almost catching up with the catalytic performance of $\mathrm{Pt} / \mathrm{C}\left(\eta_{10}=21 \mathrm{mV}\right)$, implying that the $\mathrm{Ru}$ nanoclusters serve as the main active sites. In order to compare the HER kinetics rates on the above electrocatalysts, Tafel slopes are taken from the corresponding LSV curves applying the Tafel equation [1]. In Figure 5b, Ru-pCo@NC delivers a low Tafel slope of $32.1 \mathrm{mV}$ $\mathrm{dec}^{-1}$ according to the Tafel plot depicted, which is approaching to that of $\mathrm{Pt} / \mathrm{C}(31.0 \mathrm{mV}$ $\left.\mathrm{dec}^{-1}\right)$, and much smaller than those of NC $\left(169.2 \mathrm{mV} \mathrm{dec}^{-1}\right), \mathrm{Co@NC}\left(142.9 \mathrm{mV} \mathrm{dec}^{-1}\right)$, and pCo@NC $\left(110.7 \mathrm{mV} \mathrm{dec}{ }^{-1}\right)$, implying that a faster HER reaction kinetics is achieved in $\mathrm{Ru}-\mathrm{pCo} @ \mathrm{NC}$, which can be attributed to the interaction between $\mathrm{Ru}$ and $\mathrm{O}$, as revealed by the above XPS analysis. Such a superior Tafel slope demonstrates that the HER proceeding on Ru-pCo@NC electrocatalyst follows the very efficient Volmer-Tafel mechanism, with the formation of molecular hydrogen between two adjacently adsorbed $\mathrm{H}$ atom $\left(\mathrm{H}^{*}+\mathrm{H}^{*}\right.$ $\rightarrow \mathrm{H}_{2}$ ) as the rate-determining step (RDS) [41]. Subsequently, the charge transfer resistance $\left(R_{c t}\right)$ of the electrocatalyst is evaluated by EIS. The Nyquist plots of NC, Co@NC, pCo@NC, and Ru-pCo@NC are compared in Figure 5c. As depicted, the $\mathrm{R}_{\mathrm{ct}}$ values follow the order of Ru-pCo@NC $(4.3 \Omega)<$ pCo@NC $(102.2 \Omega)<$ Co@NC $(307.6 \Omega)<\mathrm{NC}$, confirming that an extremely fast charge transfer is realized in $\mathrm{Ru}-\mathrm{pCo} @ \mathrm{NC}$, thus speeding up the reaction kinetics. Moreover, the Tafel slopes and $\mathrm{R}_{\mathrm{ct}}$ values of Ru-pCo@NC-1, Ru-pCo@NC-2, and Ru-pCo@NC-3 are also compared in Figure S6a and Figure S6b, respectively. It follows 
the same trend of their catalytic behavior, that is, first decreasing gradually and then remaining stable. The CV curves of Co@NC, pCo@NC, and Ru-pCo@NC recorded at increased scan rates (20 to $100 \mathrm{mV} \mathrm{s}^{-1}$ ) are depicted in Figure S7a-c. Accordingly, the $\mathrm{C}_{\mathrm{dl}}$ of Ru-pCo@NC is estimated to be $95.7 \mathrm{mF} \mathrm{cm}{ }^{-2}$, much higher than that of Co@NC $\left(16.4 \mathrm{mF} \mathrm{cm}^{-2}\right)$ and $\mathrm{pCo} @ \mathrm{NC}\left(36.7 \mathrm{mF} \mathrm{cm}^{-2}\right)$, meaning more exposed electrochemically active sites in Ru-pCo@NC (Figure 5d). This is as a result of its high SSA, abundant pores, and well-dispersed $\mathrm{Ru}$ active sites.
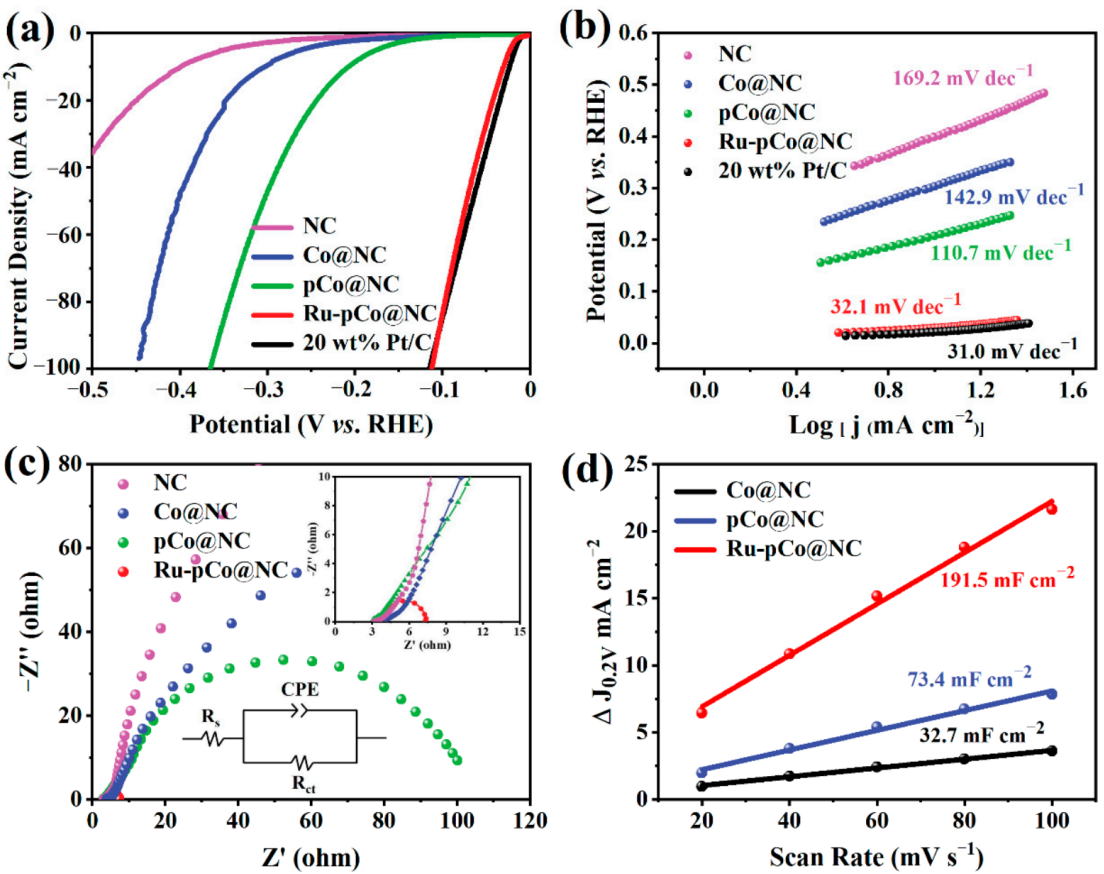

Figure 5. (a) iR-corrected LSV curves and (b) Tafel plots of NC, Co@NC, pCo@NC, Ru-pCo@NC, and $20 \mathrm{wt} \% \mathrm{Pt} / \mathrm{C}$ in $1 \mathrm{M} \mathrm{KOH}$. (c) Nyquist plots of NC, Co@NC, pCo@NC, and Ru-pCo@NC (the magnified curves and equivalent circuit are presented in the insert of c). (d) Relationship curves of capacitive current density $\left(\Delta \mathrm{J}=\left(\mathrm{J}_{\mathrm{a}}-\mathrm{J}_{\mathrm{C}}\right) / 2\right)$ at $0.2 \mathrm{~V}$ versus scan rate for the determination of $\mathrm{C}_{\mathrm{dl}} \mathrm{s}$ of Co@NC, pCo@NC, and Ru-pCo@NC.

Moreover, another crucial target for electrocatalyst in practical applications is its stability. The cycling stability of Ru-pCo@NC is evaluated by 10,000 CV cycles in $1 \mathrm{M} \mathrm{KOH}$. Significantly, the catalytic ability of Ru-pCo@NC shows negligible attenuation with the $\eta_{10}$ increasing by only $5 \mathrm{mV}$ (Figure 6a). Moreover, the long-term durability of Ru-pCo@NC is measured by chronoamperometry, and the obtained current-time $(i-t)$ curve (Figure $6 \mathrm{~b}$ ) reveals that the catalytic activity of Ru-pCo@NC could be maintained for at least $40 \mathrm{~h}$ under the alkaline condition. After durability testing, the polyhedral morphology of Ru-pCo@NC is well preserved, and Ru is still dispersed on the carbon support uniformly (Figure S8). Therefore, these results firmly manifest that the robust stability of the Ru active species and the porous carbon support.

The electrochemical performance of Ru-pCo@NC catalyst in an acid electrolyte $(0.5 \mathrm{M}$ $\mathrm{H}_{2} \mathrm{SO}_{4}$ ) is also described in Figure S9. The $\eta_{10}$ and Tafel slope of Ru-pCo@NC are $64 \mathrm{mV}$ and $69.5 \mathrm{mV} \mathrm{dec}^{-1}$, respectively (Figure S9a,b). Such performance shows slightly inferior to those of $20 \mathrm{wt} \% \mathrm{Pt} / \mathrm{C}\left(\eta_{10}=35 \mathrm{mV}, 32.4 \mathrm{mV} \mathrm{dec}^{-1}\right)$ but compare favorably with those of Co@NC $\left(\eta_{10}=451 \mathrm{mV}, 239.7 \mathrm{mV} \mathrm{dec}^{-1}\right)$ and pCo@NC $\left(\eta_{10}=258 \mathrm{mV}, 114.7 \mathrm{mV} \mathrm{dec}^{-1}\right)$, as well as most reported HER catalysts under acid condition (Table S3). The $\mathrm{R}_{\mathrm{ct}}$ of RupCo@NC determined by the Nyquist plot is $8.2 \Omega$ (Figure S9c), representing its fast electron transfer for HER in acid solution. Subsequently, the long-term stability of Ru-pCo@NC is also tested. As observed in Figure S9d, the activity of Ru-pCo@NC almost remains stable 
over testing for 15,000 s, while that of $20 \mathrm{wt} \% \mathrm{Pt} / \mathrm{C}$ shows a noticeable decay in $8000 \mathrm{~s}$, further confirming that the satisfactory stability of Ru-pCo@NC in acid solution.

(a)

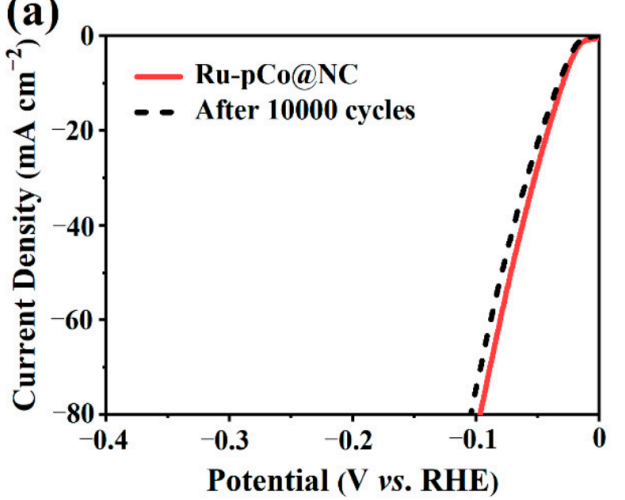

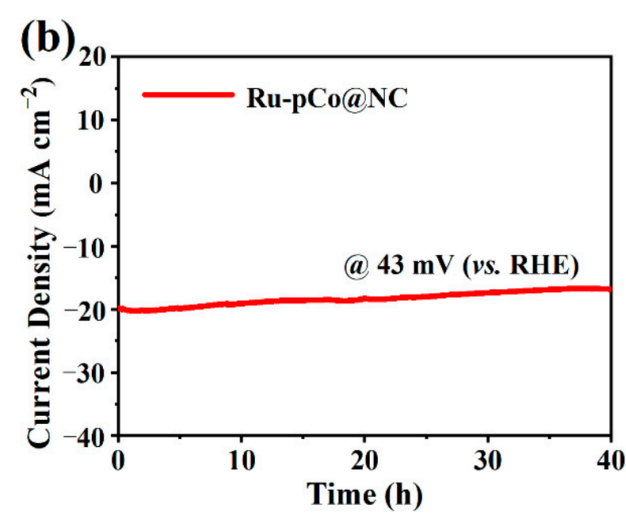

Figure 6. (a) Comparison of LSV curves for Ru-pCo@NC before and after 10000 CV cycles in $1 \mathrm{M}$ $\mathrm{KOH}$ and (b) the chronoamperometric curve of Ru-pCo@NC at a constant overpotential of $43 \mathrm{mV}$ (vs. RHE).

\section{Conclusions}

In summary, a high-performance HER electrocatalyst by introducing low-content $\mathrm{Ru}$ nanoclusters onto the $\mathrm{Co} / \mathrm{N}$-codoped porous carbon derived from a bimetallic ZIF is synthesized. Thanks to the large SSA and high porosity of carbon support, uniformly dispersed $\mathrm{Ru}$ active sites, and rapid reaction kinetics of the interaction between $\mathrm{Ru}$ and $\mathrm{O}$, the Ru-pCo@NC catalyst exhibits outstanding catalytic activity in $1 \mathrm{M} \mathrm{KOH}$ electrolyte with a low overpotential of $30 \mathrm{mV}$ at the current density of $10 \mathrm{~mA} \mathrm{~cm}^{-2}$, rivaling that of commercial Pt/C. In addition, Ru-pCo@NC also displays a desirable stability for long-time running. It is demonstrated that the small Ru nanoclusters as the main active sites in RupCo@NC play a critical role in accelerating the HER kinetics. Due to the great flexibility in the composition and structure of MOFs, this study provides a potential idea for preparing highly effective and stable HER catalysts with a favorable support structure and uniformly dispersed active sites, which is of great significance for the economic efficiency in practical applications.

Supplementary Materials: The following are available online at https://www.mdpi.com/article/ 10.3390/nano11051228/s1, Figure S1: XRD patterns of (a) as-synthesized ZIE-8, ZIF-67, ZnCo-ZIF, and simulated ZIF-67; and (b) Ru-pCo@NC-1, Ru-pCo@NC, Ru-pCo@NC-2, and Ru-pCo@NC-3. Figure S2: SEM images of (a) ZIF-8, (b) ZIF-67, (c) ZnCo-ZIF; (d) NC; (e) Co@NC; and (f) pCo@NC. Figure S3: XPS spectra of Ru-pCo@NC: (a) survey spectrum; (b) Zn 2p; and (c) Co 2p. Figure S4: $\mathrm{N}_{2}$ adsorption/desorption isotherms and (b) Raman spectra of Co@NC and pCo@NC. Figure S5: LSV curves of of Ru-pCo@NC-1, Ru-pCo@NC, Ru-pCo@NC-2, and Ru-pCo@NC-3 in 1 M KOH. Figure S6: (a) Tafel plots and (b) Nyquist plots of Ru-pCo@NC-1, Ru-pCo@NC, Ru-pCo@NC-2, and Ru-pCo@NC-3 in 1 M KOH. Figure S7: CV curves of (a) Co@NC; (b) pCo@NC; and (c) Ru-pCo@NC. Figure S8: (a) TEM image, (b) HRTEM image, and (c) HAADF-STEM and corresponding EDX elemental mapping images of Ru-pCo@NC used after chronoamperometric measurements. Figure S9: The electrochemical performance of the as-prepared catalysts in $0.5 \mathrm{M} \mathrm{H}_{2} \mathrm{SO}_{4}$ : (a) LSV curves; (b) Tafel plots; (c) Nyquist plot; and (d) chronoamperometric curve. Table S1: Comparison of Ru-based electrocatalysts towards HER in alkaline electrolyte. Table S2: Comparison of structure features for Co@NC and pCo@NC. Table S3: Comparison of the HER performance of Ru-pCo@NC with reported electrocatalysts in acidic electrolyte.

Author Contributions: Conceptualization, Y.L.; methodology, H.Q. and X.G.; software, H.Q.; validation, G.Z., F.Z., X.F., and W.P.; formal analysis, H.Q.; investigation, H.Q.; resources, Y.L.; data curation, H.Q.; writing—original draft preparation, H.Q.; writing—review and editing, G.L., M.Z., H.H., K.L., and Y.L.; visualization, H.Q.; supervision, Y.L., G.Z., F.Z., X.F., and W.P.; project administration, Y.L., 
G.Z., F.Z., X.F., and W.P.; funding acquisition, Y.L. All authors have read and agreed to the published version of the manuscript.

Funding: This research was funded by the General Program of National Natural Science Foundation of China, grant number 22078242, U20A20153, and the Program of Introducing Talents of Discipline to Universities, grant number B06006.

Institutional Review Board Statement: Not applicable.

Informed Consent Statement: Not applicable.

Data Availability Statement: The data presented in this study are available in this article and its Supplementary Materials.

Acknowledgments: This study was supported by the General Program of National Natural Science Foundation of China (Nos. 22078242, U20A20153) and the Program of Introducing Talents of Discipline to Universities (No. B06006).

Conflicts of Interest: The authors declare no conflict of interest.

\section{References}

1. Murthy, A.P.; Madhavan, J.; Murugan, K. Recent advances in hydrogen evolution reaction catalysts on carbon/carbon-based supports in acid media. J. Power Sources 2018, 398, 9-26. [CrossRef]

2. Zou, X.; Zhang, Y. Noble metal-free hydrogen evolution catalysts for water splitting. Chem. Soc. Rev. 2015, 44, 5148-5180. [CrossRef] [PubMed]

3. Balat, M. Potential importance of hydrogen as a future solution to environmental and transportation problems. Int. J. Hydrog. Energy 2008, 33, 4013-4029. [CrossRef]

4. Wen, X.; Guan, J. Recent progress on MOF-derived electrocatalysts for hydrogen evolution reaction. Appl. Mater. Today 2019, 16, 146-168. [CrossRef]

5. Kagkoura, A.; Arenal, R.; Tagmatarchis, N. Sulfur-Doped Carbon Nanohorn Bifunctional Electrocatalyst for Water Splitting. Nanomaterials 2020, 10, 2416. [CrossRef] [PubMed]

6. Guan, X.; Ma, J.; Li, K.; Liang, J.; Li, Z.; Peng, W.; Zhang, G.; Fan, X.; Zhang, F.; Li, Y. Multilevel N-doped carbon nanotube/graphene supported cobalt phosphide nanoparticles for electrocatalytic hydrogen evolution reaction. Int. J. Hydrog. Energy 2019, 44, 30053-30061. [CrossRef]

7. Lee, D.-Y.; Song, H.J.; Kim, D.-W. Wide pH range electrocatalytic hydrogen evolution using molybdenum phosphide nanoparticles uniformly anchored on porous carbon cloth. Ceram. Int. 2021, 47, 9347-9353. [CrossRef]

8. Zhu, E.; Yan, X.; Wang, S.; Xu, M.; Wang, C.; Liu, H.; Huang, J.; Xue, W.; Cai, J.; Heinz, H.; et al. Peptide-Assisted 2-D Assembly toward Free-Floating Ultrathin Platinum Nanoplates as Effective Electrocatalysts. Nano Lett. 2019, 19, 3730-3736. [CrossRef]

9. Mahmood, J.; Li, F.; Jung, S.M.; Okyay, M.S.; Ahmad, I.; Kim, S.J.; Park, N.; Jeong, H.Y.; Baek, J.B. An efficient and pH-universal ruthenium-based catalyst for the hydrogen evolution reaction. Nat. Nanotechnol. 2017, 12, 441-446. [CrossRef]

10. Zhu, L.; Lin, H.; Li, Y.; Liao, F.; Lifshitz, Y.; Sheng, M.; Lee, S.-T.; Shao, M. A rhodium/silicon co-electrocatalyst design concept to surpass platinum hydrogen evolution activity at high overpotentials. Nat. Commun. 2016, 7, 12272. [CrossRef]

11. Chen, J.; Xia, G.; Jiang, P.; Yang, Y.; Guoliang, X.; Shi, R.; Su, J.; Chen, Q. Active and Durable Hydrogen Evolution Reaction Catalyst Derived from Pd-Doped Metal-Organic Frameworks. ACS Appl. Mater. Interfaces 2016, 8, 13378-13383. [CrossRef]

12. Lim, C.S.; Sofer, Z.; Toh, R.J.; Eng, A.Y.S.; Luxa, J.; Pumera, M. Iridium- and Osmium-decorated Reduced Graphenes as Promising Catalysts for Hydrogen Evolution. ChemPhysChem 2015, 16, 1898-1905. [CrossRef]

13. Wang, J.; Wei, Z.; Mao, S.; Li, H.; Wang, Y. Highly uniform Ru nanoparticles over N-doped carbon: $\mathrm{pH}$ and temperature-universal hydrogen release from water reduction. Energy Environ. Sci. 2018, 11, 800-806. [CrossRef]

14. Su, J.W.; Yang, Y.; Xia, G.L.; Chen, J.T.; Jiang, P.; Chen, Q.W. Ruthenium-cobalt nanoalloys encapsulated in nitrogen-doped graphene as active electrocatalysts for producing hydrogen in alkaline media. Nat. Commun. 2017, 8, 10.

15. Chang, Q.B.; Ma, J.W.; Zhu, Y.Z.; Li, Z.; Xu, D.Y.; Duan, X.Z.; Peng, W.C.; Li, Y.; Zhang, G.L.; Zhang, F.B.; et al. Controllable Synthesis of Ruthenium Phosphides (RuP and RuP2) for $\mathrm{pH}-$ Universal Hydrogen Evolution Reaction, ACS Sustain. Chem. Eng. 2018, 6, 6388-6394.

16. Zong, Z.; Qian, Z.; Tang, Z.; Liu, Z.; Tian, Y.; Wang, S. Hydrogen evolution and oxygen reduction reactions catalyzed by core-shelled Fe@Ru nanoparticles embedded in porous dodecahedron carbon. J. Alloy. Compd. 2019, 784, 447-455. [CrossRef]

17. Tang, C.; Wang, H.-F.; Zhang, Q. Multiscale Principles to Boost Reactivity in Gas-Involving Energy Electrocatalysis. Acc. Chem. Res. 2018, 51, 881-889. [CrossRef]

18. Li, K.; Li, Y.; Peng, W.; Zhang, G.; Zhang, F.; Fan, X. Bimetallic Iron-Cobalt Catalysts and Their Applications in Energy-Related Electrochemical Reactions. Catalysts 2019, 9, 762. [CrossRef]

19. Li, H.; Ke, F.; Zhu, J. MOF-Derived Ultrathin Cobalt Phosphide Nanosheets as Efficient Bifunctional Hydrogen Evolution Reaction and Oxygen Evolution Reaction Electrocatalysts. Nanomaterials 2018, 8, 89. [CrossRef] 
20. Zhang, H.; Nai, J.; Yu, L.; Lou, X.W.(David). Metal-Organic-Framework-Based Materials as Platforms for Renewable Energy and Environmental Applications. Joule 2017, 1, 77-107. [CrossRef]

21. Banerjee, R.; Phan, A.; Wang, B.; Knobler, C.; Furukawa, H.; O’Keeffe, M.; Yaghi, O.M. High-Throughput Synthesis of Zeolitic Imidazolate Frameworks and Application to CO2Capture. Science 2008, 319, 939-943. [CrossRef] [PubMed]

22. He, D.; Wu, X.; Liu, W.; Lei, C.; Yu, C.; Zheng, G.; Pan, J.; Lei, L.; Zhang, X. Co1-xS embedded in porous carbon derived from metal organic framework as a highly efficient electrocatalyst for oxygen evolution reaction. Chin. Chem. Lett. 2019, 30, 229-233. [CrossRef]

23. Cheng, N.; Ren, L.; Xu, X.; Du, Y.; Dou, S.X. Recent Development of Zeolitic Imidazolate Frameworks (ZIFs) Derived Porous Carbon Based Materials as Electrocatalysts. Adv. Energy Mater. 2018, 8, 8. [CrossRef]

24. Aijaz, A.; Masa, J.; Rösler, C.; Xia, W.; Weide, P.; Botz, A.J.R.; Fischer, R.A.; Schuhmann, W.; Muhler, M. Co@Co3 O4 Encapsulated in Carbon Nanotube-Grafted Nitrogen-Doped Carbon Polyhedra as an Advanced Bifunctional Oxygen Electrode. Angew. Chem. Int. Ed. 2016, 55, 4087-4091. [CrossRef] [PubMed]

25. Yang, L.; Li, H.; Yu, Y.; Wu, Y.; Zhang, L. Assembled 3D MOF on 2D Nanosheets for Self-boosting Catalytic Synthesis of N-doped Carbon Nanotube Encapsulated Metallic Co Electrocatalysts for Overall Water Splitting. Appl. Catal. B Environ. 2020, $271,118939$. [CrossRef]

26. Jiang, H.-L.; Liu, B.; Lan, Y.-Q.; Kuratani, K.; Akita, T.; Shioyama, H.; Zong, F.; Xu, Q. From Metal-Organic Framework to Nanoporous Carbon: Toward a Very High Surface Area and Hydrogen Uptake. J. Am. Chem. Soc. 2011, 133, 11854-11857. [CrossRef]

27. Meng, J.; Niu, C.; Xu, L.; Li, J.; Liu, X.; Wang, X.; Wu, Y.; Xu, X.; Chen, W.; Li, Q.; et al. General Oriented Formation of Carbon Nanotubes from Metal-Organic Frameworks. J. Am. Chem. Soc. 2017, 139, 8212-8221. [CrossRef]

28. Yu, J.; Chen, G.; Sunarso, J.; Zhu, Y.; Ran, R.; Zhu, Z.; Zhou, W.; Shao, Z. Cobalt Oxide and Cobalt-Graphitic Carbon Core-Shell Based Catalysts with Remarkably High Oxygen Reduction Reaction Activity. Adv. Sci. 2016, 3, 1600060. [CrossRef]

29. Liu, X.; Jin, T.(Leo); Hood, Z.D.; Tian, C.; Guo, Y.; Zhan, W. Mechanochemically Assisted Synthesis of Ruthenium Clusters Embedded in Mesoporous Carbon for an Efficient Hydrogen Evolution Reaction. ChemElectroChem 2019, 6, 2719-2725. [CrossRef]

30. Yuan, K.; Lützenkirchen-Hecht, D.F.; Li, L.; Shuai, L.; Li, Y.; Cao, R.; Qiu, M.; Zhuang, X.; Leung, M.K.H.; Chen, Y.; et al Boosting Oxygen Reduction of Single Iron Active Sites via Geometric and Electronic Engineering: Nitrogen and Phosphorus Dual Coordination. J. Am. Chem. Soc. 2020, 142, 2404-2412. [CrossRef]

31. Chen, D.; Lu, R.; Pu, Z.; Zhu, J.; Li, H.-W.; Liu, F.; Hu, S.; Luo, X.; Wu, J.; Zhao, Y.; et al. Ru-doped 3D flower-like bimetallic phosphide with a climbing effect on overall water splitting. Appl. Catal. B Environ. 2020, 279, 119396. [CrossRef]

32. Kim, M.; Ju, H.; Kim, J. Dihydrogen phosphate ion functionalized nanocrystalline thallium ruthenium oxide pyrochlore as a bifunctional electrocatalyst for aqueous Na-air batteries. Appl. Catal. B Environ. 2019, 245, 29-39. [CrossRef]

33. Duan, X.; Zhou, J.; Qian, G.; Li, P.; Zhou, X.; Chen, D. Carbon Nanofiber-Supported Ru Catalysts for Hydrogen Evolution by Ammonia Decomposition. Chin. J. Catal. 2010, 31, 979-986. [CrossRef]

34. Greeley, J.; Jaramillo, T.F.; Bonde, J.; Chorkendorff, I.B.; Nørskov, J.K. Computational high-throughput screening of electrocatalytic materials for hydrogen evolution. Nat. Mater. 2006, 5, 909-913. [CrossRef]

35. Ma, J.; Wang, M.; Lei, G.; Zhang, G.; Zhang, F.; Peng, W.; Fan, X.; Guoliang, Z. Polyaniline Derived N-Doped Carbon-Coated Cobalt Phosphide Nanoparticles Deposited on N-Doped Graphene as an Efficient Electrocatalyst for Hydrogen Evolution Reaction. Small 2018, 14, 14. [CrossRef]

36. Zhao, M.; Li, K.; Gong, N.; Guan, X.; He, H.; Qi, H.; Peng, J.; Peng, W.; Zhang, G.; Fan, X.; et al. Ni modified ultrafine MoxC (x = 1, 2) wrapped by nitrogen-doped carbon for efficient hydrogen evolution reaction in acid and alkaline electrolytes. Int. J. Hydrog. Energy 2020, 45, 28285-28293. [CrossRef]

37. Callejas, J.F.; Read, C.G.; Popczun, E.J.; McEnaney, J.M.; Schaak, R.E. Nanostructured Co2P Electrocatalyst for the Hydrogen Evolution Reaction and Direct Comparison with Morphologically Equivalent CoP. Chem. Mater. 2015, 27, 3769-3774. [CrossRef]

38. Su, Y.; Zhu, Y.; Jiang, H.; Shen, J.; Yang, X.; Zou, W.; Chen, J.; Li, C. Cobalt nanoparticles embedded in N-doped carbon as an efficient bifunctional electrocatalyst for oxygen reduction and evolution reactions. Nanoscale 2014, 6, 15080-15089. [CrossRef]

39. Sathiskumar, C.; Ramakrishnan, S.; Vinothkannan, M.; Kim, A.R.; Karthikeyan, S.; Yoo, D.J. Nitrogen-Doped Porous Carbon Derived from Biomass Used as Trifunctional Electrocatalyst toward Oxygen Reduction, Oxygen Evolution and Hydrogen Evolution Reactions. Nanomaterials 2019, 10, 76. [CrossRef]

40. Li, X.; Duan, X.; Han, C.; Fan, X.; Li, Y.; Zhang, F.; Zhang, G.; Peng, W.; Wang, S. Chemical activation of nitrogen and sulfur co-doped graphene as defect-rich carbocatalyst for electrochemical water splitting. Carbon 2019, 148, 540-549. [CrossRef]

41. Guo, T.; Wang, L.; Sun, S.; Wang, Y.; Chen, X.; Zhang, K.; Zhang, D.; Xue, Z.; Zhou, X. Layered MoS2@graphene functionalized with nitrogen-doped graphene quantum dots as an enhanced electrochemical hydrogen evolution catalyst. Chin. Chem. Lett. 2019, 30, 1253-1260. [CrossRef] 